\title{
Magnaporthe effectors on the move
}

\begin{abstract}
A study in Plant Cell has begun to reveal the details of the secretion and cell-to-cell movement of Magnaporthe oryzae effector proteins.

M. oryzae is the causative agent of rice blast disease. Infection of rice begins with the attachment of a spore to the leaf cuticle. After spore germination, the germ tube differentiates into an appressorium, which matures and can then penetrate the cuticle. During the resultant biotrophic stage, the primary invasive hypha, surrounded by an extra-invasive hyphal membrane,
\end{abstract}

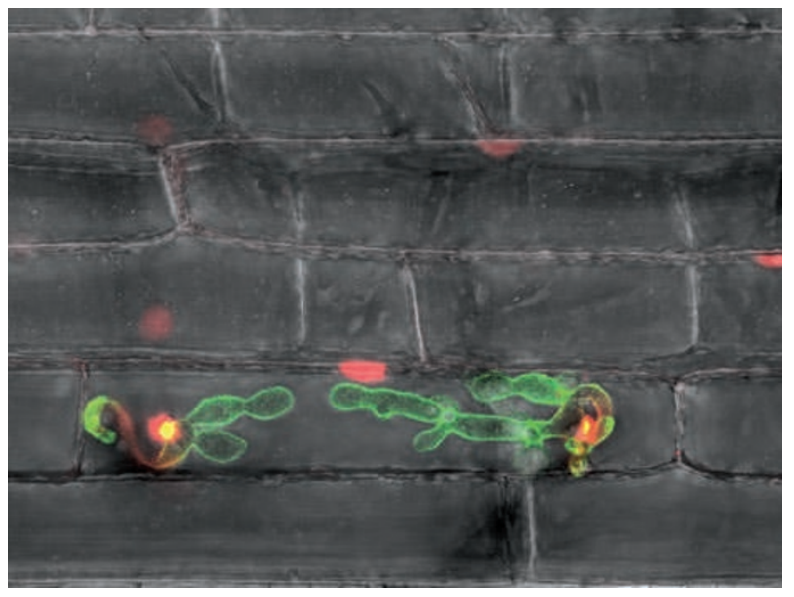

Projected confocal microscopy image of Magnaporthe oryzae secreting an effector protein, Pwl2, linked to a red fluorescent protein and a nuclear localization signal, and a putative interfacial matrix protein, Bas4, linked to green fluorescent protein. Pwl2 preferentially accumulated in the biotrophic interfacial complex (yellow). PwI2, but not Bas4, was translocated into the rice cytoplasm, shown by the concentration of the red signal in the host nucleus. After reaching the cytoplasm, Pwl 2 moved ahead into adjoining non-invaded cells. Image courtesy of C. H. Khang and B. V., Kansas State University, Manhattan, Kansas, USA. differentiates into a bulbous invasive hypha, which grows within the epidermal cell. Successful biotrophic invasion requires the release of effector proteins, which are thought to be crucial for M. oryzae to suppress plant defences. Although some effector proteins have been identified, few details on their secretion and translocation are available.

Barbara Valent and colleagues fused $M$. oryzae effector proteins to various fluorescent-protein constructs and studied their movements during $M$. oryzae infection of rice using live-cell imaging. The effectors AVR-Pita1, Pwll and Pwl2 were found to be secreted by invasive hyphae and to accumulate in a novel structure the authors call the biotrophic interfacial complex (BIC). Detailed observations revealed that this structure develops from the membranous cap (a membrane-rich extension of the extra-invasive hyphal membrane at the primary hyphal tip) and remains adjacent to the first differentiated bulbous invasive hyphal cell. What regulates effector protein accumulation in the BIC? In general, fungal effectors do not contain the highly conserved translocation motifs that are found in effectors from other eukaryotic pathogens, such as Phytophthora spp. and Plasmodium falciparum. Instead, the authors found that the region encompassing the promoters and signal peptide-encoding sequences of effector-encoding genes was sufficient to confer the preferential accumulation of effectors in the BIC.

They next looked at translocation from the BIC into the rice cell cytoplasm. Two effectors, Pwl2 and Bas1, were detected not only in the first invaded cell but also in neighbouring cells that had not been invaded, and the authors conclude that it is likely that the movement of M. oryzae effectors into non-invaded cells occurs through the plasmodesmata. It has previously been proposed that $M$. oryzae invasive hyphae might secrete signals to prepare adjacent cells for invasion, and this is supported by the fact that these effectors could be detected up to four cells ahead of the first invaded cell.

This work has provided the first detailed insight into the movements of $M$. oryzae effectors in rice. Further research is required to determine the precise mechanisms involved.

Sheilagh Molloy

ORIGINAL RESEARCH PAPER Khang, C. H. et al. Translocation of Magnaporthe oryzae effectors into rice cells and their subsequent cellto-cell movement. Plant Cell 30 Apr 2010 (doi: 10.1105/tpc.109.069666)

FURTHER READING Wilson, R. A. \& Talbot, N. J. Under pressure: investigating the biology of plant infection by Magnaporthe oryzae. Nature Rev. Microbiol. 7, 185-195 (2009) 